\title{
Modulation of inhibitory systems to enhance motor rehabilitation: insights for the use of noninvasive brain stimulation
}

\author{
Jay S. Reidler, Bruno C. Nascimento, Daniel S.K. Wu, Marcelo Carvas, Breno K. Massuyama, \\ Bruno M.R. de Oliveira, Soroush Zaghi, Daniel T. de Rezende, Rasheda El-Nazer, Walyson N. \\ Gonçalves, Lotfi B. Merabet, and Felipe Fregni \\ Harvard University, Boston, MA, USA
}

\begin{abstract}
Motor impairment following stroke is a leading cause of disability in adults. Despite advances in motor rehabilitation techniques, many adult stroke survivors never approach full functional recovery. Intriguingly, children exhibit better rehabilitation outcomes when compared to adults suffering from comparable brain injuries, yet the reasons for this remain unclear. A common explanation is that neuroplasticity in adults is substantially limited following stroke, thus constraining the brain's ability to reorganize in response to neurological insult. This explanation, however, does not suffice for there is much evidence suggesting that neuroplasticity in adults is not limited following stroke. We hypothesize that diminished functional recovery in adults is in part due to inhibitory neuronal interactions, such as transcallosal inhibition, that serve to optimize motor performance as the brain matures. Following stroke, these inhibitory interactions pose rigid barriers to recovery by inhibiting activity in the affected regions and hindering recruitment of compensatory pathways. In contrast, children exhibit better rehabilitation outcomes in part because they have not fully developed the inhibitory interactions that impede functional recovery in adults. We suggest that noninvasive brain stimulation can be used in the context of motor rehabilitation following stroke to reduce the effects of existing inhibitory connections, effectively returning the brain to a state that is more amenable to rehabilitation. We conclude by discussing further research to explore this hypothesis and its implications. Keywords: stroke, motor recovery, plasticity, rehabilitation, transcranial magnetic stimulation, transcranial direct current stimulation.
\end{abstract}

Received 27 January 2010; received in revised form 15 October 2010; accepted 30 October 2010. Available on line 28 December 2010

\section{Introduction}

Functional recovery following stroke poses a significant challenge. Current rehabilitation strategies adopt multidisciplinary approaches that include physical, occupational, cognitive, and other forms of therapy. The Bobath approach, for instance, is a widely utilized technique

Jay S. Reidler, Bruno C. Nascimento, Daniel S.K. Wu, Marcelo Carvas, Breno K. Massuyama, Bruno M.R. de Oliveira, Soroush Zaghi, Daniel T. de Rezende, Rasheda El-Nazer, Walyson N. Gonçalves, and Felipe Fregni, Laboratory of Neuromodulation, Spaulding Rehabilitation Hospital, Harvard Medical School, Boston, USA. Soroush Zaghi and Felipe Fregni, Berenson-Allen Center for Noninvasive Brain Stimulation, Beth Israel Deaconess Medical Center, Harvard Medical School, Boston, USA. Lotfi B. Merabet, Massachusetts Eye and Ear Infirmary, Harvard Medical School, Boston, USA. Correspondence regarding this article should be directed to: Felipe Fregni, MD, PhD; Laboratory of Neuromodulation, Spaulding Rehabilitation Hospital, 125 Nashua Street \#727, Boston, MA 02114. Phone: + 1-617-5732195. E-mail: ffregni@bidmc.harvard.edu that aims to integrate postural control with task performance and to train coordinated sequences of movement for a given task (Graham, Eustace, Brock, Swain, \& IrwinCarruthers, 2009; Natarajan et al., 2008). Although this and other rehabilitation techniques have reduced morbidity and institutionalized care, functional outcomes remain limited (Desrosiers, Bourbonnais, Corriveau, Gosselin, \& Bravo, 2005; Hendricks, van Limbeek, Geurts, \& Zwarts, 2002; Indredavik, Bakke, Slordahl, Rokseth, \& Haheim, 1999; Langhammer \& Stanghelle, 2003). Indeed, motor impairment following stroke is a leading cause of disability in adults, with long term medical expenses resulting from stroke representing a majority of costs associated with this expensive disease (Demaerschalk, Hwang, \& Leung, 2010). It is estimated that less than $15 \%$ of stroke patients exhibiting paralysis in the upper or lower extremities achieve complete motor recovery (Hendricks et al., 2002).

While functional improvement is limited in adults, interestingly, children suffering from comparable brain injuries exhibit substantially better rehabilitation outcomes. Following stroke, children often demonstrate 
relatively minor long-term motor deficits (Hariman, Griffith, Hurtig, \& Keehn, 1991) as compared to adults (Hendricks et al., 2002). Overall functional outcomes appear to be better in children than in adults (Kim, Han, \& Kim, 2009), and studies suggest that normative function is achieved by over $35 \%$ of pediatric stroke patients (deVeber, MacGregor, Curtis, \& Mayank, 2000) compared to less than $25 \%$ of adult patients (Hendricks et al., 2002). Moreover, following hemispherectomy, children exhibit remarkable functional recovery that is generally superior to that observed in adults (Benecke, Meyer, \& Freund, 1991; Gardner, Karnosh, McClure, \& Gardner, 1955; Kossoff, Buck, \& Freeman, 2002).

The biological underpinnings for these improved outcomes in children remain unclear, and their elucidation has the potential to further our understanding of poststroke impairment and rehabilitation. One hypothesis to explain the limited outcomes in adults compared to children is that synaptic plasticity in the adult brain is substantially limited following stroke, thus constraining its ability to reorganize in response to neurological insult. This explanation is contradicted, however, by a large body of evidence suggesting that plasticity in the adult brain is not limited following stroke (Calautti \& Baron, 2003; Cramer \& Chopp, 2000; Jones, Kleim, \& Greenough, 1996; Jones \& Schallert, 1992; Stroemer, Kent, \& Hulsebosch, 1998).

We hypothesize that functional recovery in adults is relatively limited not because of an inherent lack of brain plasticity, but rather because of inhibitory neuronal interactions, such as transcallosal inhibition, that serve to optimize motor performance as the brain matures (Nowak, Grefkes, Ameli, $\&$ Fink, 2009). Following stroke, these inhibitory interactions hinder rehabilitation by inhibiting activity in the affected regions and impeding recruitment of compensatory pathways (Murase, Duque, Mazzocchio, \& Cohen, 2004; PascualLeone, Amedi, Fregni, \& Merabet, 2005). Children, however, exhibit relatively better rehabilitation outcomes in part because they have not fully developed the inhibitory interactions that impede functional recovery in adults (Heinen et al., 1998; Mall et al., 2004; Moll et al., 1999).

In this paper, we will discuss evidence supporting this hypothesis and potential methods for improving motor recovery by focusing on modulating interhemispheric interactions and cortical excitability of lesioned areas using novel methods of noninvasive brain stimulation. Noninvasive brain stimulation can, for instance, be used in the context of motor rehabilitation following stroke to reduce the effects of existing inhibitory connections, effectively returning the brain to a state that is more amenable to rehabilitation.

\section{Neuroplasticity following stroke: evidence at the molecular, cellular and systemic levels}

As mentioned above, one explanation for the relatively low functional recovery in adults is that neuroplasticity might be reduced in the brain following stroke. Here, we present evidence on the molecular, cellular, and systemic levels that suggests that neuroplasticity is not generally limited following stroke.

At the molecular level, studies have shown that following ischemic insult, there is a re-emergence of developmental proteins that are otherwise normally absent or present at very low concentrations (Cramer \& Chopp, 2000; Stroemer et al., 1998). These proteins are associated with increased neuroplasticity and include differentiation factors (e.g., NeuroD), structural proteins (e.g., Nestin, MAP-2), growth associated proteins (e.g., GAP43, synaptophysin), as well as growth factors (e.g., BFGF, VEGF, BDNF) (Cramer \& Chopp, 2000). Interestingly, these proteins are increased not only at the local site of injury, but in distant areas as well. Furthermore, while these proteins re-emerge within hours to days following the lesion, they remain effective for weeks and months after the initial insult (Cramer \& Chopp, 2000).

At the cellular level, there is also convincing evidence of increased plasticity following injury. One study in adult rats demonstrated that the propensity for long-term potentiation is increased in perilesional areas after experimentally induced focal cortical infarction (Hagemann, Redecker, Neumann-Haefelin, Freund, \& Witte, 1998). Similarly, unilateral injury to the sensorimotor cortex in adult rats has been shown to induce dramatic growth of neuronal dendrites in the contralateral homotopic cortex (Jones et al., 1996). Finally, neocortical injury has been shown to increase dendritic branching (Jones \& Schallert, 1992) and synaptic density (Jones et al., 1996; Stroemer et al., 1998). These studies suggest that on both molecular and cellular levels, mechanisms geared toward promoting brain reorganization are enhanced, rather than inhibited, following injury.

At the systemic level, evidence suggests that dramatic cortical reorganization also takes place following stroke. A study in adult primates, for instance, found cortical remapping along the margins of the site of focal damage (Xerri, Merzenich, Peterson, \& Jenkins, 1998). Furthermore, following focused rehabilitative training of skilled movements, the motor area representation of the impaired hand in primates have been shown to extend to areas formerly associated with shoulder and elbow movements (Nudo, Wise, SiFuentes, \& Milliken, 1996). Consistent with the animal findings above, motor areas associated with finger movements in adult stroke patients have been shown to be remapped onto areas traditionally associated with face motor control (Weiller, Ramsay, Wise, Friston, \& Frackowiak, 1993). Another study in similar patients found that recovery from stroke was associated with motor cortical reorganization including bihemisphereic recruitment of premotor cortical areas (Seitz et al., 1998).

Studies in adult patients using functional magnetic resonance imaging (fMRI) and positron emission 
tomography (PET) provide robust evidence for cortical reorganization following stroke. A longitudinal fMRI study of 20 first-time adult stroke survivors, for instance, showed task-related changes in brain activation over the course of recovery (Ward, Brown, Thompson, \& Frackowiak, 2003). An extensive review of PET and fMRI studies supports the conclusion that the damaged adult brain is indeed capable of reorganizing as a means of compensating for motor impairments (Calautti \& Baron, 2003).

This cortical remapping has also been observed in healthy adults receiving low frequency $(1 \mathrm{~Hz})$ repetitive transcranial magnetic stimulation (rTMS) delivered to the contralateral primary motor cortex (M1) (Lee et al., 2003). rTMS is a method of noninvasive brain stimulation that uses a large, rapidly changing magnetic field to induce electrical stimulating currents in the brain. At low frequency of stimulation, this procedure is presumed to decrease neuronal firing and excitability (Pascual-Leone et al., 2005; Pascual-Leone et al., 1998). Lee et al. (2003) found that subjects who performed simple motor tasks following rTMS of the motor cortex showed sustained motor function while having activation of secondary motor areas such as the rostral supplementary motor cortex, ipsilateral primary motor cortex, and dorsal premotor cortex (as detected by fMRI). They suggest that this rTMS-induced remapping may be similar to the acute compensatory plasticity of the motor system following stroke.

The above human and animal studies strongly support the notion that neuroplasticity is present in adults following stroke. While the studies do not provide direct comparisons to children, they do counter the claim that neuroplasticity is absent in adults and call into question whether differences in neuroplasticity alone can explain the poor functional recovery in adults following stroke.

\section{Alternative neural pathways in stroke recovery}

Alternative neural pathways, such as corticospinal projections from the ipsilesional dorsolateral premotor cortex (PMd) and supplementary motor area (SMA), appear to play important roles in stroke recovery. These projections are known to be less numerous and less effective at exciting spinal cord motor neurons when compared to projections from the primary motor cortex (Maier et al., 2002). However, several studies have shown that these alternative neural pathways are recruited in stroke recovery. For instance, there are reports of increased ipsilesional PMdactivation associated with therapy-induced improvement in upper limb function (Johansen-Berg et al., 2002) and gait function (Miyai et al., 2003). Furthermore, chronic stroke patients exhibit simple task impairments when the ipsilesional PMd is disrupted by TMS (a finding not observed in healthy controls), demonstrating again the importance of this alternative neural pathway in motor recovery (Fridman et al., 2004).
While alternative neural pathways appear to play an important role in motor recovery following stroke, their role remains limited. Indeed, long-term motor recovery is more strongly associated with the extent and location of damage to direct corticospinal projections from the primary motor cortex, with greater preservation and activation of direct corticospinal tract following stroke predicting better functional outcomes in patients (Cruz Martinez, Tejada, \& Diez Tejedor, 1999; Pennisi et al., 1999). Longitudinal studies have shown that enhanced motor recovery in adults is associated with cortical activation patterns more similar to those observed before stroke, suggesting that optimum recovery following stroke requires a shift from initial compensatory patterns of activation, using alternative neural pathways to prestroke patterns using the affected primary motor cortex (Jang et al., 2004; Jang et al., 2003; Ward et al., 2003).

In summary, while remapping to alternative neural pathways appears important for motor recovery following strokes, better results are achieved in patients who are able to eventually shift neural activity back to its pre-stroke state. It seems reasonable to infer that anatomical preservation of motor neural systems is important for such outcomes. Interestingly, this does not appear to be the case for children recovering from brain lesions. Studies have shown that even in extremes cases such as hemispherectomy, younger patients achieve significantly greater functional recovery than older subjects (Benecke et al., 1991; Gardner et al., 1955). Controlled animal studies support the conclusion that younger age predicts better outcomes as well. For instance, a study comparing motor outcomes in neonatal and adult hemispherectomized cats, found that the neonatal group relearned how to use their impaired limbs following one practice session while the adult group needed almost eight training sessions to acquire the same motor skill (Burgess, Villablanca, \& Levine, 1986). A recent experiment directly comparing hemispherectomy in infant and adult monkeys similarly showed better functional outcomes in the younger animals (Burke, Zangenehpour, \& Ptito, 2010). As discussed above, these differences in outcomes across age cannot simply be due to an utter lack of neuroplasticity in older subjects, as neuroplasticity and recruitment of alternative neural pathways have been widely observed in adults. A deeper understanding of the effects of age on brain function, injury, and recovery may provide useful insight into these differences.

\section{Maturation and inhibitory systems: age as an important determinant of stroke recovery}

Maturation of the nervous system is a complex process involving elaborate formation, myelination, and elimination (pruning) of synaptic connections that starts in the uterus and extends into teenage years (Garvey 
\& Mall, 2008; Huttenlocher \& de Courten, 1987). With respect to motor function, maturation includes the development of handedness, loss of synkinesis (by age 10), and gradual increase in motor performance over time (Frye, Rotenberg, Ousley, \& Pascual-Leone, 2008; Garvey \& Mall, 2008; Huttenlocher, 1979; Huttenlocher \& de Courten, 1987). One interesting example of maturational processes associated with the motor system is the development of the corticospinal system and its relation with shorter central conduction time and enhanced motor performance. When assessed at different ages, motor latency decreases with age while skillfulness increases, likely due to enhanced synaptic connectivity and increased myelination and axonal growth (Fietzek et al., 2000).

Enhanced motor performance during maturation reflects not only the development of excitatory connections, but also the formation and consolidation of inhibitory interactions across the nervous system. Throughout maturation, interhemispheric inhibitory connections across the corpus callosum develop that result in activated primary motor cortical areas inhibiting homologous areas in the contralateral primary motor cortex, known as transcallosal inhibition (Nowak et al., 2009). This inhibition refines movement by, for instance, inhibiting activity in the nonactive hand during activity of the active hand.

Transcallosal inhibition is commonly observed by using TMS to detect cortical silent periods (CSP), or periods of depressed activity following cortical activation. Specifically, transcallosal inhibition results in ipsilateral CSPs, wherein activation of the contralateral motor cortex by TMS results in activity of the ipsilateral hand and silent periods in the ipsilateral motor cortex. Ipsilateral silent period is thought to be produced by excitatory callosal neurons projected to the inhibitory interneurons of the contralateral motor cortex (Ferbert et al., 1992). As measured by ipsilateral CSPs, transcallosal inhibition has been found to be completely absent in preschool children under the age of six, only reaching adult levels in children around ten years of age (Heinen et al., 1998; Moll et al., 1999). Transcallosal inhibition appears to play a role in inhibiting ipsilateral corticospinal projections, which are detected in two-thirds of children under 10, but not in adults (Muller, Kass-Iliyya, \& Reitz, 1997). In addition to developing interhemispheric inhibition throughout maturation, children also appear to develop intracortical inhibition (ICI) as they mature. Indeed, ICI is nearly 4 times greater in adults when compared to children less than 10 (Mall et al., 2004). Interestingly, these combined studies are consistent with findings that myelination of the intracortical and callosal white matter is only completed by early adulthood (Garvey \& Mall, 2008). In sum, maturation during childhood involves the development of interhemispheric and intracortical inhibitory synaptic connections, which have the net effect of refining motor function.
While inhibitory neural connections enhance motor performance throughout maturation, they appear to have maladaptive effects in the context of motor recovery following stroke. Following stroke, the intact hemisphere exerts transcallosal inhibition on the affected recovering hemisphere (Murase et al., 2004). This leads to decreased cortical excitability in the affected cortex relative to the unaffected cortex, thereby impairing the neuroplastic processes necessary for achieving full functional recovery (Pascual-Leone, 2005). Indeed the amount of transcallosal inhibition of the affected cortex is positively associated with the severity of the functional impairment of the affected hand (Murase et al., 2004).

The observation that transcallosal inhibition hinders motor rehabilitation, may in part explain the better functional outcomes in pediatric patients as compared to adults following stroke and other forms of brain injury. As discussed above, children below the age of 10 have not fully developed the interhemispheric and intracortical inhibitory systems that impede full motor recovery (Heinen et al., 1998; Mall et al., 2004). Therefore, cortical excitability in their lesioned cortices will have greater cortical excitability than their adult counterparts, making them more amenable to the neuroplastic processes necessary for recruiting alternative neural pathways, returning to pre-stroke activation patterns, and achieving full functional recovery.

The suggestion that relatively low inhibitory drive in younger patients facilitates recovery may shed light on the remarkable functional outcomes observed in children following brain injury. As previously mentioned, research confirms that pediatric stroke survivors and neonatal hemispherectomy patients surpass their adult counterparts in outcome measures (Benecke et al., 1991; Gardner et al., 1955; Kim et al., 2009). One study comparing patients hemispherectomized before and after brain maturation found that TMS stimulation evoked faster and stronger ipsilateral compound muscle action potentials in patients that underwent hemispherectomy prior to brain maturation (Benecke et al., 1991). This study suggests that maturation of inhibitory systems not only renders alternative neural pathways less accessible in adulthood, but also less efficient, with slower and weaker responses than those observed in children. Conversely, the combined results of these studies suggest that the relatively reduced inhibitory systems in children facilitate neuroplastic changes and recruitment of compensatory pathways.

The above discussion suggests that diminished functional recovery in adults is in part due to inhibitory neuronal interactions, such as transcallosal inhibition, that serve to optimize motor performance as the brain matures. Following stroke, these inhibitory interactions pose rigid barriers to recovery by inhibiting activity in the affected regions and hindering recruitment of compensatory pathways. Building off of this 
understanding, we next discuss noninvasive brain stimulation techniques that aim to modulate these inhibitory systems, thereby returning the brain to a state that is more amenable to rehabilitation.

\section{Using noninvasive brain stimulation to guide brain plasticity in stroke recovery}

Despite the many rehabilitative therapies that are currently employed, functional recovery following stroke remains fairly limited. Based on the above discussion, we propose the use of noninvasive brain stimulation, particularly transcranial magnetic stimulation (TMS) and transcranial direct current stimulation (tDCS), as techniques for modulating inhibitory systems that predominate in adults and for enhancing motor recovery following stroke.

The use of electro-magnetic brain stimulation has been investigated since the late $19^{\text {th }}$ century (Walsh, Pascual-Leone, \& Kosslyn, 2005). It was not until the mid 1980's, however, that TMS was introduced, when Anthony Barker and colleagues solved the technical challenges involved in penetrating the scalp and skull with a sufficiently strong and quick magnetic field pulse (Barker, Jalinous, \& Freeston, 1985). Whereas tDCS (discussed below) is considered to be a purely neuromodulatory technique, TMS is a neurostimulatory and neuromodulatory technique. TMS uses the principle of electromagnetic induction to produce localized, induced currents in the brain (Hallett, 2000). These currents can be of sufficient magnitude to depolarize neurons and stimulate action potentials, and when these currents are applied repetitively, as in repetitive transcranial magnetic stimulation (rTMS), they can also modulate cortical excitability beyond the time of stimulation, either decreasing or increasing general excitability (depending on the parameters of stimulation; Pascual-Leone et al., 1999).

tDCS is a less expensive and more portable technique that involves the transmission of low amplitude direct currents from sponge electrodes through the skull and to the underlying cortex. Although there is substantial shunting of current at the scalp, studies have shown that sufficient current penetrates the brain to modify the transmembrane resting potentials of cortical neurons and thereby influence their firing rate and general excitability in response to input (Miranda, Lomarev, \& Hallett, 2006; Wagner et al., 2007). When tDCS is applied for a sufficient duration, it has after-effects on neuronal spontaneous activity as demonstrated in humans by shifts in cortical excitability that can be observed well beyond the period of stimulation (Nitsche \& Paulus, 2001).

Below we suggest three ways in which noninvasive brain stimulation can be used to enhance patient care and motor recovery following stroke. First, we discuss the potential use of TMS as a diagnostic tool for recording neurophysiological measures and tracking improvement following stroke. We next discuss how rTMS and tDCS can be used to counteract maladaptive consequences of inhibitory systems and excite affected cortical areas, thereby facilitating motor recovery. Finally, we discuss how tDCS can be used to enhance synaptic plasticity and re-learning of motor skills following stroke.

\section{Transcranial magnetic stimulation as a diagnostic tool}

When applied to the motor cortex at a suitable intensity, TMS induces the depolarization of pyramidal cells, generating a descending corticospinal volley that depolarizes spinal motoneurons and results in the induction of a muscle contraction. The amplitude of the muscle contraction (motor evoked potential; MEP) is a measure of the activation of the motor cortex at a specific point in time. In addition, applied to motor and non-motor brain regions, TMS induces depolarization of cortical neurons and activates cortico-cortical and cortico-subcortical connections that induce specific changes in brain activity, which can be recorded using EEG. Such measures can provide insights into brain connectivity both within and across hemispheres. Importantly, the impact of TMS depends on the level of local cortical excitability and thus on the balance of intracortical facilitation and inhibition present in the brain. Therefore, neurophysiological measures obtained using TMS measures can provide unique insights regarding conditions in which there are imbalances in intracortical excitability, such as in stroke or brain damage.

Besides the assessment of motor evoked potential and motor threshold, which measure global corticospinal excitability levels, various measures of intra- and intercortical inhibition and facilitation are also possible with TMS. These include: (1) short interval intracortical inhibition (SICI) (Kujirai et al., 1993). It is assumed that SICI involves the inhibition of action potentials resulting from the second, supra-threshold pulse by the activation of low-threshold inhibitory circuits (via inhibitory post synaptic potentials; IPSP) generated by the first, sub-threshold pulse. It is widely believed that SICI is GABA $_{A}$-dependent since GABA agonists increase SICI (Ziemann, Rothwell, \& Ridding, 1996); (2) long interval intracortical inhibition (LICI), where both TMS pulses are delivered at supra-threshold intensities with an interstimulus interval of 50-200 ms. There is strong evidence that LICI is mediated by long-lasting $\mathrm{GABA}_{\mathrm{B}}$ dependent IPSPs and activation of pre-synaptic $\mathrm{GABA}_{\mathrm{B}}$ receptors on inhibitory interneurons (Werhahn, Kunesch, Noachtar, Benecke, \& Classen, 1999); (3) intracortical facilitation (ICF), the amplitude of a test MEP can be enhanced if it is accompanied by a sub-threshold conditioning pulse applied 10-25 ms earlier (Kujirai et al., 1993). Glutamatergic interneurons at the level of M1 are likely to be involved in ICF since it is reduced by NMDA antagonists such as dextromethorphan; and (4) transcallosal inhibition, in which the two motor cortices 
are stimulated with a delay of $10 \mathrm{~ms}$. The first pulse (the conditioning pulse) is applied over the primary motor cortex and the second pulse (the test pulse) is applied after a delay of $10 \mathrm{~ms}$ in the contralateral primary motor cortex. It has been shown that the second pulse is associated with a significant inhibition in the MEP characteristics.

As discussed above, transcallosal inhibition begins developing in children 6 years of age (Heinen et al., 1998) and appears to have maladaptive effects in the context of motor recovery following stroke (Murase et al., 2004).

These neurophysiological parameters can give a relatively accurate estimate of the level of inhibition in the motor systems. TMS can therefore serve as a diagnostic tool to monitor the natural progression of stroke and measure the effects of therapies on recovery.

rTMS and tDCS to modulate maladaptive inhibitory systems and increase motor recovery

As discussed in this review, inhibitory interactions are strengthened during maturation, which limit the ability of the cortex to recruit alternative neural pathways. In fact, after stroke there are mutifocal, bihemispheric changes in brain activity, which pose rigid barriers to recovery by inhibiting activity in the affected regions and hindering recruitment of compensatory pathways (Ward et al., 2003). An example here is the increased transcallosal inhibition that is observed in the acute phases following stroke (Murase et al., 2004).

Numerous investigations point to the conclusion that activation of the motor cortex is of paramount importance to promote motor recovery. Anatomically, it is the most common source of fibers for the pyramidal tract (Galea \& Darian-Smith, 1994), which is key to generating rapid, distal, or fractionated movements (Brinkman \& Kuypers, 1973). Studies in animals (Dijkhuizen et al., 2003) and humans (Marshall et al., 2000) have described a hemispheric shift in sensorimotor cortex activation from bilateral to stroke-affected hemisphere in association with post-stroke recovery, particularly among patients with subcortical stroke (Feydy et al., 2002; Theoret, Kobayashi, Valero-Cabre, \& Pascual-Leone, 2003).

Following stroke of the motor cortex, cortical excitability is relatively decreased as compared to the unaffected motor cortex (Shimizu et al., 2002) possibly due to a shift in interhemispheric interactions with increased transcallosal inhibition from the unaffected to the affected motor cortex (Murase et al., 2004). Similar shifts in interhemispheric interactions have been postulated across parietal cortices in strokes leading to neglect syndrome (Kinsbourne, 1977).

Modulation of interhemispheric competition using rTMS has been demonstrated in normal subjects. For example, low frequency rTMS can improve motor performance with the ipsilateral hand by releasing interhemispheric inhibition in the unstimulated hemisphere (Kobayashi, Hutchinson,
Theoret, Schlaug, \& Pascual-Leone, 2004). Similarly, low frequency rTMS of one parietal cortex improves ipsilateral attention by enhancing excitability in the unstimulated parietal cortex (Hilgetag, et al., 2001). In the setting of a stroke, there might thus be desirable effects induced by either suppressing activity in the undamaged hemisphere or increasing activity in the perilesional cortex of the damaged hemisphere, thereby promoting a restoration of activity across bihemispheric neural networks and guidance of more adaptive plasticity (Fregni \& Pascual-Leone, 2006; Hummel \& Cohen, 2006).

Indeed, preliminary studies using rTMS to enhance motor recovery in stroke patients provide promising results. In a sham-controlled trial that included patients up to 12 months following stroke, Mansur and colleagues (2005) applied 0.5 Hz rTMS to the unaffected hemisphere for 10 minutes to suppress cortical activity and maladaptive transcallosal inhibition influencing the affected hemisphere. They found that the treatment significantly improved performance of simple and choice reaction time tests as well as the Purdue Pegboard test in the affected hand (Mansur et al., 2005). Extending these findings to children, a randomized study in 10 pediatric patients ( $>7$ years old) who suffered from subcortical arterial ischaemic stroke found that contralesional inhibitory rTMS seemed to improve hand function in children with hemiparesis, with no serious adverse effects (Kirton et al., 2008).

Preliminary studies using tDCS to modulate motor cortex activity in stroke patients and healthy subjects have also returned favorable results. Fregni et al. (2005b) examined whether reduction of activity in the contralesional hemispheres of stroke patients by introducing cathodal (inhibitory) tDCS would result in improved motor performance due to decreased transcallosal inhibition. They found that cathodal stimulation of the contralesional hemisphere, as well as anodal (excitatory) stimulation of the ipsilesional hemisphere, significantly improved motor performance compared to sham tDCS (Fregni et al., 2005a). In a recent sham-controlled investigation of healthy subjects, Williams, Pascual-Leone, and Fregni (2010) combined bilateral motor cortex tDCS with contralateral hand restraint of the dominant hand. When comparing active- to sham- stimulation, they found a decrease in cortical excitability in the dominant hemisphere and a decrease in transcallosal inhibition from the dominant hemisphere to the non-dominant hemisphere. Moreover, this decrease in transcallosal inhibition was positively associated with motor performance enhancement in the non-dominant hand (Williams et al., 2010).

In sum, the above discussion suggests that both rTMS and tDCS may be useful tools for decreasing maladaptive interhemispheric inhibition, exciting lesioned cortex, and facilitating motor recovery following stroke. 
Using tDCS to enhance the effects of cognitivebehavioral interventions

Following stroke, activation of neural networks that support re-learning of motor skills remains an important challenge. While physical therapy may be a valuable approach to achieving motor recovery, it is of limited efficacy when administered alone. Here we discuss how tDCS can be used to enhance the efficacy and efficiency of cognitive-behavioral interventions by promoting neuroplasticity and consolidation of newly formed synaptic connections.

The acquisition of new skills and behaviors is linked to changes in neuronal activity and excitability (Facchini, Muellbacher, Battaglia, Boroojerdi, \& Hallett, 2002). This might reflect changes in synaptic strength, for example, through N-methyl-D-aspartate (NMDA) receptor-dependent long-term potentiation (LTP) (Rioult-Pedotti, Friedman, Hess, \& Donoghue, 1998). Successful manipulation of cortical excitability to improve learning processes and cognitive training has been demonstrated in humans in neuropharmacological investigations (Butefisch et al., 2002), with TMS (Butefisch, Khurana, Kopylev, \& Cohen, 2004), and with deafferentation of adjacent or contralateral body parts (Facchini et al., 2002; Floel et al., 2004). tDCS thus presents an interesting alternative because it is non-invasive, painless (as compared to TMS and deafferentation), and without serious side effects (as compared to neuropharmacological agents). In addition, tDCS has an important theoretical advantage as it directly modifies spontaneous neuronal activity and therefore can increase activity in a more physiological manner. tDCS also offers a valuable practical advantage as investigators and study subjects can be reliably blinded, therefore allowing for well-controlled experiments (Gandiga, Hummel, \& Cohen, 2006).

Nitsche and colleagues showed that anodal tDCS of the human motor cortex elicits prolonged cortical excitability increases (Nitsche \& Paulus, 2000, 2001), most likely by inducing sub-threshold neuronal membrane depolarization (Purpura \& McMurtry, 1965). Moreover, it has been shown that tDCS evokes aftereffects that are NMDA receptor dependent (Liebetanz, Nitsche, Tergau, \& Paulus, 2002) and that share some similarity with the LTP and LTD presumed to underlie learning processes (Rioult-Pedotti et al., 1998). These studies suggest that anodal tDCS has the potential to improve rehabilitative learning by focally increasing cortical excitability and modulating NMDA receptor dependent plasticity in areas that facilitate motor recovery during behavioral training.

Indeed studies suggest that tDCS enhances motor learning in healthy subjects (Boggio et al., 2006) and stroke patients (Fregni \& Pascual-Leone, 2005; Hummel $\&$ Cohen, 2005). These findings have been confirmed by others and extended to language function in normal subjects
(Floel \& Cohen, 2007) and in patients with aphasia (Monti et al., 2008). There are also preliminary reports in healthy subjects of improved verbal fluency (Iyer et al., 2005) and verbal working memory abilities (Fregni, 2005b) associated with combined tDCS/behavioral training as compared to behavioral training alone. Thus tDCS may serve as a simple yet powerful supplementary tool for enhancing learning associated with physical rehabilitation (Bolognini, Pascual-Leone, \& Fregni, 2009).

\section{Conclusions}

Functional motor recovery following stroke remains limited, with many adult stroke survivors never approaching full functional recovery. Interestingly, children exhibit better rehabilitation outcomes when compared to adults suffering from comparable brain injuries, though the reasons why remain unclear. Here, we discuss possible biological explanations for the inadequate functional recovery commonly observed in adults as well as the remarkable recoveries commonly observed in children.

It is clear from evidence on the molecular, cellular, and systemic levels that neuroplasticity is not absent in adult patients following stroke, and, to the contrary, that a multitude of mechanisms geared toward promoting brain reorganization are enhanced following injury. It is therefore unlikely that an inherent lack of neuroplasticity alone could fully account for relatively poor functional recovery in adults. We suggest that decreased motor recovery in adults is in part due to inhibitory neuronal interactions, such as transcallosal inhibition, that serve to optimize motor performance as the brain matures. Following stroke, these inhibitory interactions pose rigid barriers to recovery by inhibiting activity in the affected regions and hindering recruitment of compensatory pathways. We suggest that children exhibit better rehabilitation outcomes in part because they have not fully developed the inhibitory interactions that obstruct functional recovery in adults.

We conclude with a discussion of how TMS and tDCS can be used to enhance patient care and motor recovery following stroke. TMS can be used as a diagnostic tool for recording neurophysiological measures and tracking improvement following stroke. Both techniques can be employed to effectively restore interhemispheric balance to the brain by inhibiting the inhibitory systems that develop as one ages, effectively creating brain states that might be partially equivalent to a "premature" state. Both techniques can also enhance synaptic plasticity and relearning of motor skills following stroke. In fact we have recently compared intensive motor training with sham tDCS vs. intensive motor training with tDCS aimed to reduce transcallosal inhibition from the unaffected to the affected motor cortex. In this study we have observed that intensive motor training alone has inferior results as compared to the group that also received tDCS to 
decrease transcallosal inhibition. Indeed the additional beneficial behavioral effects were correlated to a decrease in transcallosal inhibition (data not published).

Additional research is needed to support the conclusions we discuss here. While we present a large literature that demonstrates robust neuroplasticity in adults, more direct comparisons quantifying the extent of neuroplasticity in children compared to adults (on molecular, cellular, and systemic levels) would be needed to rule out the possibility that differences in neuroplasticity across age can account for rehabilitation differences. Furthermore, a properly controlled experiment in healthy subjects could be proposed to examine our hypothesis that poor functional recovery in adult stroke patients compared to pediatric stroke patients is largely due to maladaptive consequences of increased transcallosal inhibition in adults. Such an experiment might involverTMS induction of intracortical inhibition and facilitation in adults and kids as they learn a motor task, and measuring how transcallosal inhibition correlates with motor learning throughout the experiment. Future studies looking at the effects of rTMS and tDCS on motor recovery following stroke, should combine these noninvasive brain stimulation techniques with different rehabilitation approaches, employ varying parameters, and utilize greater numbers of subjects, so that we can reliably evaluate these techniques and generate optimal rehabilitative therapies.

\section{Acknowledgement}

This publication was made possible with partial funding by grant number R21 7R21DK081773 from the National Institute of Diabetes and Digestive and Kidney Diseases (NIDDK) at the National Institutes of Health. Its contents are solely the responsibility of the authors and do not necessarily represent the official views of NIDDK.

\section{References}

Barker, A. T., Jalinous, R., \& Freeston, I. L. (1985). Non-invasive magnetic stimulation of human motor cortex. Lancet, 1, 1106-1107.

Benecke, R., Meyer, B. U., \& Freund, H. J. (1991). Reorganisation of descending motor pathways in patients after hemispherectomy and severe hemispheric lesions demonstrated by magnetic brain stimulation. Experimental Brain Research, 83(2), 419-426.

Boggio, P. S., Castro, L. O., Savagim, E. A., Braite, R., Cruz, V. C., Rocha, R. R.,... Fregni, F. (2006). Enhancement of nondominant hand motor function by anodal transcranial direct current stimulation. Neurosci Lett, 404, 232-236.

Bolognini, N., Pascual-Leone, A., \& Fregni, F. (2009). Using noninvasive brain stimulation to augment motor training-induced plasticity. Journal of Neuroengeneering and Rehabilitation, 6, 8.

Brinkman, J., \& Kuypers, H. G. (1973). Cerebral control of contralateral and ipsilateral arm, hand and finger movements in the split-brain rhesus monkey. Brain, 96, 653-674.

Burgess, J. W., Villablanca, J. R., \& Levine, M. S. (1986). Recovery of functions after neonatal or adult hemispherectomy in cats. III. Complex functions: open field exploration, social interactions, maze and holeboard performances. Behavioural Brain Research, 20(2), 217-230.

Burke, M. W., Zangenehpour, S., \& Ptito, M. (2010). Partial recovery of hemiparesis following hemispherectomy in infant monkeys. Neuroscience Letters, 469, 243-247.

Butefisch, C. M., Davis, B. C., Sawaki, L., Waldvogel, D., Classen, J., Kopylev, L., \& Cohen, L.G. (2002). Modulation of use-dependent plasticity by d-amphetamine. Annals of Neurology, 51(1), 59-68.

Butefisch, C. M., Khurana, V., Kopylev, L., \& Cohen, L. G. (2004). Enhancing encoding of a motor memory in the primary motor cortex by cortical stimulation. Journal of Neurophysiology, 95, 2110-2116.

Calautti, C., \& Baron, J. C. (2003). Functional neuroimaging studies of motor recovery after stroke in adults: a review. Stroke, 34, 1553-1566.

Cramer, S. C., \& Chopp, M. (2000). Recovery recapitulates ontogeny. Trends in Neurosciences, 23, 265-271.

Cruz Martinez, A., Tejada, J., \& Diez Tejedor, E. (1999). Motor hand recovery after stroke. Prognostic yield of early transcranial magnetic stimulation. Electroencephalography and Clinical Neurophysiology, 39, 405-410.

Demaerschalk, B. M., Hwang, H. M., \& Leung, G. (2010). US cost burden of ischemic stroke: a systematic literature review. American Journal of Manage Care, 16, 525-533.

Desrosiers, J., Bourbonnais, D., Corriveau, H., Gosselin, S., \& Bravo, G. (2005). Effectiveness of unilateral and symmetrical bilateral task training for arm during the subacute phase after stroke: a randomized controlled trial. Clinical Rehabilitation, 19, 581-593.

deVeber, G. A., MacGregor, D., Curtis, R., \& Mayank, S. (2000). Neurologic outcome in survivors of childhood arterial ischemic stroke and sinovenous thrombosis. Journal Child Neurolology, 15, 316-324.

Dijkhuizen, R. M., Singhal, A. B., Mandeville, J. B., Wu, O., Halpern, E. F., Finklestein, S. P.,...Lo, E.H. (2003). Correlation between brain reorganization, ischemic damage, and neurologic status after transient focal cerebral ischemia in rats: a functional magnetic resonance imaging study. Journal of Neuroscience, 23(2), 510-517.

Facchini, S., Muellbacher, W., Battaglia, F., Boroojerdi, B., \& Hallett, M. (2002). Focal enhancement of motor cortex excitability during motor imagery: a transcranial magnetic stimulation study. Acta Neurological Scandinavica, 105, 146-151.

Ferbert, A., Priori, A., Rothwell, J. C., Day, B. L., Colebatch, J. G., \& Marsden, C. D. (1992). Interhemispheric inhibition of the human motor cortex. Journal of Physiology, 453, 525-546.

Feydy, A., Carlier, R., Roby-Brami, A., Bussel, B., Cazalis, F., Pierot, L.,...Mayer, M.A. (2002). Longitudinal study of motor recovery after stroke: recruitment and focusing of brain activation. Stroke, 33, 1610-1617.

Fietzek, U. M., Heinen, F., Berweck, S., Maute, S., Hufschmidt, A., Schulte-Monting, J., ...Korinthenberg, R. (2000). Development of the corticospinal system and hand motor function: central conduction times and motor performance tests. Developmental Medicine Child Neurology,42,220-227.

Floel, A., \& Cohen, L. G. (2007). Contribution of noninvasive cortical stimulation to the study of memory functions. Brain Research Review, 53, 250-259.

Floel, A., Nagorsen, U., Werhahn, K. J., Ravindran, S., Birbaumer, N.,...Cohen, L.G. (2004). Influence of somatosensory input on motor function in patients with chronic stroke. Annals of Neurololy, $56,206-212$.

Fregni, F., Boggio, P. S., Mansur, C. G., Wagner, T., Ferreira, M. J., Lima, M. C.,... Pascual-Leone, A. (2005a). Transcranial direct current stimulation of the unaffected hemisphere in stroke patients. Neuroreport, 16, 1551-1555.

Fregni, F., Boggio, P. S., Nitsche, M., Bermpohl, F., Antal, A., Feredoes,...Pascual-Leone, A. (2005b). Anodal transcranial direct current stimulation of prefrontal cortex enhances working memory. Experimental Brain Research, 166(1), 23-30.

Fregni, F., \& Pascual-Leone, A. (2005). Transcranial magnetic stimulation for the treatment of depression in neurologic disorders. Current Psychiatry Report, 7, 381-390.

Fregni, F., \& Pascual-Leone, A. (2006). Hand motor recovery after stroke: tuning the orchestra to improve hand motor function. Cognitive and Behavioral Neurology, 19(1), 21-33.

Fridman, E. A., Hanakawa, T., Chung, M., Hummel, F., Leiguarda, R. C., \& Cohen, L. G. (2004). Reorganization of the human ipsilesional premotor cortex after stroke. Brain, 127(Pt 4), 747-758.

Frye, R. E., Rotenberg, A., Ousley, M., \& Pascual-Leone, A. (2008). Transcranial magnetic stimulation in child neurology: current and future directions. Journal Child of Neurology, 23(1), 79-96.

Galea, M. P., \& Darian-Smith, I. (1994). Multiple corticospinal neuron 
populations in the macaque monkey are specified by their unique cortical origins, spinal terminations, and connections. Cerebral Cortex, 4, 166-194.

Gandiga, P. C., Hummel, F. C., \& Cohen, L. G. (2006). Transcranial DC stimulation (tDCS): a tool for double-blind sham-controlled clinical studies in brain stimulation. Clinical Neurophysiology, $117,845-850$.

Gardner, W. J., Karnosh, L. J., McClure, C. C., Jr., \& Gardner, A. K. (1955). Residual function following hemispherectomy for tumour and for infantile hemiplegia. Brain, 78, 487-502.

Garvey, M. A., \& Mall, V. (2008). Transcranial magnetic stimulation in children. Clinical Neurophysiology, 119, 973-984.

Graham, J. V., Eustace, C., Brock, K., Swain, E., \& Irwin-Carruthers, S. (2009). The Bobath concept in contemporary clinical practice. Top Stroke Rehabilitation, 16(1), 57-68.

Hagemann, G., Redecker, C., Neumann-Haefelin, T., Freund, H. J., \& Witte, O. W. (1998). Increased long-term potentiation in the surround of experimentally induced focal cortical infarction. Annals of Neurology, 44, 255-258.

Hallett, M. (2000). Transcranial magnetic stimulation and the human brain. Nature, 406, 147-150.

Hariman, L. M., Griffith, E. R., Hurtig, A. L., \& Keehn, M. T. (1991). Functional outcomes of children with sickle-cell disease affected by stroke. Archives of Physical Medicine and Rehabilitation, 72, 498-502.

Heinen, F., Glocker, F. X., Fietzek, U., Meyer, B. U., Lucking, C. H., \& Korinthenberg, R. (1998). Absence of transcallosal inhibition following focal magnetic stimulation in preschool children. Annals of Neurology, 43, 608-612.

Hendricks, H. T., van Limbeek, J., Geurts, A. C., \& Zwarts, M. J. (2002). Motor recovery after stroke: a systematic review of the literature. Archives of Physical Medicine and Rehabilitation, 83, 1629-1637.

Hilgetag, C. C., Theoret, H., \& Pascual-Leone, A. (2001). Enhanced visual spatial attention ipsilateral to rTMS-induced 'virtual lesions' of human parietal cortex. Nature Neuroscience, 4, 953-957.

Hummel, F., \& Cohen, L. G. (2005). Improvement of motor function with noninvasive cortical stimulation in a patient with chronic stroke. Neurorehabil Neural Repair, 19(1), 14-19.

Hummel, F. C., \& Cohen, L. G. (2006). Non-invasive brain stimulation: a new strategy to improve neurorehabilitation after stroke? Lancet Neurology, 5, 708-712

Huttenlocher, P. R. (1979). Synaptic density in human frontal cortex - developmental changes and effects of aging. Brain Research, 163(2), 195-205

Huttenlocher, P. R., \& de Courten, C. (1987). The development of synapses in striate cortex of man. Human Neurobiology, 6(1), 1-9.

Indredavik, B., Bakke, F., Slordahl, S. A., Rokseth, R., \& Haheim, L. L. (1999). Stroke unit treatment. 10-year follow-up. Stroke, 30, 1524-1527.

Iyer, M. B., Mattu, U., Grafman, J., Lomarev, M., Sato, S., \& Wassermann, E. M. (2005). Safety and cognitive effect of frontal DC brain polarization in healthy individuals. Neurology, 64, 872-875.

Jang, S. H., Cho, S. H., Kim, Y. H., Kwon, Y. H., Byun, W. M., Lee, S. J., et al. (2004). Cortical activation changes associated with motor recovery in patients with precentral knob infarct. Neuroreport, 15, 395-399.

Jang, S. H., Kim, Y. H., Cho, S. H., Lee, J. H., Park, J. W., \& Kwon, Y. H. (2003). Cortical reorganization induced by task-oriented training in chronic hemiplegic stroke patients. Neuroreport, 14(1), 137-141.

Johansen-Berg, H., Dawes, H., Guy, C., Smith, S. M., Wade, D. T., \& Matthews, P. M. (2002). Correlation between motor improvements and altered fMRI activity after rehabilitative therapy. Brain, $125(\mathrm{Pt}$ 12), 2731-2742.

Jones, T. A., Kleim, J. A., \& Greenough, W. T. (1996). Synaptogenesis and dendritic growth in the cortex opposite unilateral sensorimotor cortex damage in adult rats: a quantitative electron microscopic examination. Brain Research, 733(1), 142-148.

Jones, T. A., \& Schallert, T. (1992). Overgrowth and pruning of dendrites in adult rats recovering from neocortical damage. Brain Research, 581(1), 156-160.

Kim, C. T., Han, J., \& Kim, H. (2009). Pediatric stroke recovery: a descriptive analysis. Archives of Physical Medicine and Rehabilitation, 90, 657-662.

Kinsbourne, M. (1977). Hemi-neglect and hemisphere rivalry. Advanced Neurology, 18, 41-49.

Kirton, A., Chen, R., Friefeld, S., Gunraj, C., Pontigon, A. M., \& Deveber, G. (2008). Contralesional repetitive transcranial magnetic stimulation for chronic hemiparesis in subcortical paediatric stroke: a randomised trial. Lancet Neurology, 7, 507-513

Kobayashi, M., Hutchinson, S., Theoret, H., Schlaug, G., \& Pascual-Leone, A. (2004). Repetitive TMS of the motor cortex improves ipsilateral sequential simple finger movements. Neurology, 62(1), 91-98.

Kossoff, E. H., Buck, C., \& Freeman, J. M. (2002). Outcomes of 32 hemispherectomies for Sturge-Weber syndrome worldwide. Neurology, 59, 1735-1738.

Kujirai, T., Caramia, M. D., Rothwell, J. C., Day, B. L., Thompson, P. D., Ferbert, A., et al. (1993). Corticocortical inhibition in human motor cortex. Journal of Physiology, 471, 501-519.

Langhammer, B., \& Stanghelle, J. K. (2003). Bobath or motor relearning programme? A follow-up one and four years post stroke. Clinical Rehabilitation, 17, 731-734.

Lee, L., Siebner, H. R., Rowe, J. B., Rizzo, V., Rothwell, J. C., Frackowiak, R. S.,... Friston, K.J. (2003). Acute remapping within the motor system induced by low-frequency repetitive transcranial magnetic stimulation. Journal of Neuroscience, 23, 5308-5318

Liebetanz, D., Nitsche, M. A., Tergau, F., \& Paulus, W. (2002). Pharmacological approach to the mechanisms of transcranial DC-stimulation-induced after-effects of human motor cortex excitability. Brain, 125(Pt 10), 2238-2247.

Maier, M. A., Armand, J., Kirkwood, P. A., Yang, H. W., Davis, J. N., \& Lemon, R. N. (2002). Differences in the corticospinal projection from primary motor cortex and supplementary motor area to macaque upper limb motoneurons: an anatomical and electrophysiological study. Cerebral Cortex, 12, 281-296.

Mall, V., Berweck, S., Fietzek, U. M., Glocker, F. X., Oberhuber, U., Walther, M., ...Heinen, F. (2004). Low level of intracortical inhibition in children shown by transcranial magnetic stimulation. Neuropediatrics, 35, 120-125.

Mansur, C. G., Fregni, F., Boggio, P. S., Riberto, M., Gallucci-Neto, J., Santos, C. M.,...Pascual-Leone, A.(2005). A sham stimulationcontrolled trial of rTMS of the unaffected hemisphere in stroke patients. Neurology, 64, 1802-1804.

Marshall, R. S., Perera, G. M., Lazar, R. M., Krakauer, J. W., Constantine, R. C., \& DeLaPaz, R. L. (2000). Evolution of cortical activation during recovery from corticospinal tract infarction. Stroke, 31, 656-661.

Miranda, P. C., Lomarev, M., \& Hallett, M. (2006). Modeling the current distribution during transcranial direct current stimulation. Clinical Neurophysiology, 117, 1623-1629.

Miyai, I., Yagura, H., Hatakenaka, M., Oda, I., Konishi, I., \& Kubota, K. (2003). Longitudinal optical imaging study for locomotor recovery after stroke. Stroke, 34, 2866-2870.

Moll, G. H., Heinrich, H., Wischer, S., Tergau, F., Paulus, W., \& Rothenberger, A. (1999). Motor system excitability in healthy children: developmental aspects from transcranial magnetic stimulation. Electroencephalography and Clinical Neurophysiology (Suppl), 51, 243-249.

Monti, A., Cogiamanian, F., Marceglia, S., Ferrucci, R., Mameli, F., Mrakic-Sposta, S.,... Priori, A. (2008). Improved naming after transcranial direct current stimulation in aphasia. Journal of Neurology Neurosurgery Psychiatry, 79, 451-453.

Muller, K., Kass-Iliyya, F., \& Reitz, M. (1997). Ontogeny of ipsilateral corticospinal projections: a developmental study with transcranial magnetic stimulation. Annals of Neurology, 42, 705-711.

Murase, N., Duque, J., Mazzocchio, R., \& Cohen, L. G. (2004). Influence of interhemispheric interactions on motor function in chronic stroke. Annals of Neurology, 55, 400-409.

Natarajan, P., Oelschlager, A., Agah, A., Pohl, P. S., Ahmad, S. O., \& Liu, W. (2008). Current clinical practices in stroke rehabilitation: regional pilot survey. Journal of Rehabilitation Research and Development, 45, 841-849.

Nitsche, M. A., \& Paulus, W. (2000). Excitability changes induced in the human motor cortex by weak transcranial direct current stimulation. Journal of Physiology, 527 (Pt 3), 633-639.

Nitsche, M. A., \& Paulus, W. (2001). Sustained excitability elevations induced by transcranial DC motor cortex stimulation in humans. Neurology, 57, 1899-1901.

Nowak, D. A., Grefkes, C., Ameli, M., \& Fink, G. R. (2009). Interhemispheric competition after stroke: brain stimulation to enhance recovery of function of the affected hand. Neurorehabilitation Neural Repair, 23, 641-656.

Nudo, R. J., Wise, B. M., SiFuentes, F., \& Milliken, G. W. (1996). Neural substrates for the effects of rehabilitative training on motor recovery after ischemic infarct. Science, 272, 1791-1794. 
Pascual-Leone, A., Amedi, A., Fregni, F., \& Merabet, L. B. (2005). The plastic human brain cortex. Annual Review Neuroscience, 28, 377-401.

Pascual-Leone, A., Tarazona, F., Keenan, J., Tormos, J. M., Hamilton, R., \& Catala, M. D. (1999). Transcranial magnetic stimulation and neuroplasticity. Neuropsychologia, 37, 207-217.

Pascual-Leone, A., Tormos, J. M., Keenan, J., Tarazona, F., Canete, C., \& Catala, M. D. (1998). Study and modulation of human cortical excitability with transcranial magnetic stimulation. Journal of Clinical Neurophysiology, 15, 333-343.

Pennisi, G., Rapisarda, G., Bella, R., Calabrese, V., Maertens De Noordhout, A., \& Delwaide, P. J. (1999). Absence of response to early transcranial magnetic stimulation in ischemic stroke patients: prognostic value for hand motor recovery. Stroke, 30, 2666-2670.

Purpura, D. P., \& McMurtry, J. G. (1965). Intracellular Activities and Evoked Potential Changes During Polarization of Motor Cortex. Journal of Neurophysiology, 28, 166-185.

Rioult-Pedotti, M. S., Friedman, D., Hess, G., \& Donoghue, J. P. (1998). Strengthening of horizontal cortical connections following skill learning. Nature Neuroscience, 1, 230-234.

Seitz, R. J., Hoflich, P., Binkofski, F., Tellmann, L., Herzog, H., \& Freund, H. J. (1998). Role of the premotor cortex in recovery from middle cerebral artery infarction. Archives of Neurology, 55, 1081-1088.

Shimizu, T., Hosaki, A., Hino, T., Sato, M., Komori, T., Hirai, S., \& Rossini, P.M.(2002). Motor cortical disinhibition in the unaffected hemisphere after unilateral cortical stroke. Brain, 125(Pt 8), 1896-1907.

Stroemer, R. P., Kent, T. A., \& Hulsebosch, C. E. (1998). Enhanced neocortical neural sprouting, synaptogenesis, and behavioral recovery with D-amphetamine therapy after neocortical infarction in rats. Stroke, 29, 2381-2393; discussion 2393-2385.
Theoret, H., Kobayashi, M., Valero-Cabre, A., \& Pascual-Leone, A. (2003). Exploring paradoxical functional facilitation with TMS. Suppl Clinical Neurophysiology, 56, 211-219.

Wagner, T., Fregni, F., Fecteau, S., Grodzinsky, A., Zahn, M., \& Pascual-Leone, A. (2007). Transcranial direct current stimulation: a computer-based human model study. Neuroimage, 35, 1113-1124.

Walsh, V., Pascual-Leone, A., \& Kosslyn, S.M. . (2005). Transcranial Magnetic Stimulation : A Neurochronometrics of Mind. Cambrigde, MA: The MIT Press.

Ward, N. S., Brown, M. M., Thompson, A. J., \& Frackowiak, R. S. (2003). Neural correlates of motor recovery after stroke: a longitudinal fMRI study. Brain, 126(Pt 11), 2476-2496.

Weiller, C., Ramsay, S. C., Wise, R. J., Friston, K. J., \& Frackowiak, R. S. (1993). Individual patterns of functional reorganization in the human cerebral cortex after capsular infarction. Annals of Neurology, 33(2), 181-189.

Werhahn, K. J., Kunesch, E., Noachtar, S., Benecke, R., \& Classen, J. (1999). Differential effects on motorcortical inhibition induced by blockade of GABA uptake in humans. J Physiol, 517 ( Pt 2), 591-597.

Williams, J. A., Pascual-Leone, A., \& Fregni, F. (2010). Interhemispheric modulation induced by cortical stimulation and motor training. Physical Therapy, 90, 398-410.

Xerri, C., Merzenich, M. M., Peterson, B. E., \& Jenkins, W. (1998). Plasticity of primary somatosensory cortex paralleling sensorimotor skill recovery from stroke in adult monkeys. Journal of Neurophysiology, 79, 2119-2148.

Ziemann, U., Rothwell, J. C., \& Ridding, M. C. (1996). Interaction between intracortical inhibition and facilitation in human motor cortex. Journal of Physiology, 496 (Pt 3), 873-881. 